Proceedings of the 2007 Winter Simulation Conference

S. G. Henderson, B. Biller, M.-H. Hsieh, J. Shortle, J. D. Tew, and R. R. Barton, eds.

\title{
BI-CRITERIA EVALUATION OF AN OUTPATIENT PROCEDURE CENTER VIA SIMULATION
}

\author{
Todd R. Huschka \\ Division of Health Care Policy \& Research \\ 200 First St. SW, Mayo Clinic \\ Rochester, MN, 55905, U.S.A.
}

Brian T. Denton

Edward P. Fitts Department of

Industrial \& Systems Engineering

North Carolina State University

Raleigh, NC 27695-7906

\author{
Serhat Gul \\ John W. Fowler \\ Department of Industrial Engineering \\ Arizona State University \\ Tempe, AZ 85281, U.S.A.
}

\begin{abstract}
Surgical services require the coordination of many activities, including patient check-in and surgical preparation, surgery, and recovery after surgery. Each of these activities requires the availability of resources including staff, operating rooms, and intake and recovery beds. Furthermore, each of these activities has substantial uncertainty in their duration. The combination of a complex resource constrained environment, and uncertainty in the duration of activities, creates challenging scheduling problems. In this study we report on a discrete event simulation model of an outpatient surgical suite, and investigate the impact of several sequencing and scheduling heuristics on competing performance criteria.
\end{abstract}

\section{INTRODUCTION}

Surgical services require the coordination of many activities. Typically the demand for surgery occurs at the end of a stream of referrals, beginning with a patient's primary care provider, and possibly including visits to several specialists before the need for surgery is identified. Therefore, there can be considerable uncertainty in the type and number of surgeries to be scheduled on a particular day. Furthermore, recent trends in surgery indicate a rising proportion of surgeries are performed on an outpatient basis. Such surgeries tend to be shorter in duration than inpatient surgeries, and also have shorter intake and recovery times. Combining the high volume of activities in an outpatient surgical suite each day with uncertainty in the durations of such activities presents challenging scheduling problems for surgical suite directors.
Outpatient surgical suites have multiple operating rooms, with a variety of supporting resources, such as nurses, nurse anesthetists, surgeons, intake rooms, recovery rooms, and various kinds of diagnostic equipment and surgical instrument kits. Surgical procedures occur in three major stages. Intake begins when the patient arrives on the day of surgery to initiate the check-in process and ends with the patient being taken to an Operating Room (OR). Intra-operative care is defined as the time between when the patient reaches the OR bed and the time they are admitted to the recovery area. Recovery is the time between admission to the recovery area and the time that the patient is discharged from the recovery area. Even for very routine surgery (e.g. endoscopy) the time for intake, surgery, and recovery exhibits considerable uncertainty in duration (Denton, Rahman, Nelson, and Bailey 2006).

It is common for some ORs to be specialized for certain types of surgery. In such cases, specialized equipment is dedicated to the OR. In contrast, when ORs are not specialized, mobile equipment may be moved from one OR to another. Some examples of specialized equipment include diagnostic imaging equipment for gastrointestinal endoscopy, specialized surgical instruments, and cardiopulmonary bypass equipment for coronary interventions. A parallel also exists with respect to the nursing staff, who often have specialized training. The level of cross-training of staff has a significant effect on the ability to generate feasible OR schedules. The specialized environment, staff, and equipment resources increase the challenge of scheduling surgery.

There are a number of performance measures that surgical suite directors may consider in relation to the efficiency of a surgical suite including: OR and recovery room utiliza- 


\section{Huschka, Denton, Gul and Fowler}

tion, surgeon and nurse idle time, patient waiting time, and late closure of the surgical suite. These measures are not independent of each other and often are in conflict with each other. For example, patient waiting time is generally negatively correlated with surgeon idle time. Thus, the operation of a surgical suite is a multi-criteria problem. Furthermore, high fixed costs and the above mentioned factors combine to make for a highly constrained scheduling environment.

In this paper we describe a discrete event simulation model for a newly designed surgical suite at Mayo Clinic in Rochester, MN. We discuss the unique design aspects of the suite, describe the development and validation of the simulation model, and present numerical results illustrating daily operational aspects of the surgical suite. With regard to the numerical results, we investigate the impact of scheduling heuristics and daily surgical mix on performance measures. The remainder of the article is organized as follows. In the next section, we present a brief literature review of relevant simulation studies. In Section 3 provide background on the surgical scheduling process. In Section 4 we describe the simulation model and in Section 5 we discuss several sequencing and scheduling heuristics that we evaluate. In section 6 we present numerical results illustrating the effects of sequencing and scheduling heuristics and surgical mix on multiple criteria. Finally, in section 7 we summarize the key findings and resulting managerial insights.

\section{LITERATURE REVIEW}

Simulation models of operating rooms can serve several purposes, including the analysis of surgical suite design, evaluation of resource utilization, and the analysis and comparison of scheduling methods. Following is a brief review that covers several examples from the literature of previous simulation studies of surgical suites.

Dexter and Marcon (2006) studied the impact of several different surgery sequencing heuristics on workload of a post anesthesia care unit (PACU) including: Random Sequence, Longest Cases First (LCF), Shortest Cases First (SCF), Johnson's rule, and several others. The authors analyzed how sequencing affects OR over-utilization, PACU completion time, delays in discharging from the OR into PACU, and the maximum number of patients in PACU throughout the day. The simulation model in their study consists of three stages. In the first stage, cases are generated and assigned to ORs based on a utilization threshold of $70 \%$. In the second stage, case durations are simulated using their scheduled OR time multiplied by a random variable. In the third and final stage, cases are rebalanced among ORs as they are completed. Thus, if an OR completes all of its cases for the day, the cases on the waiting list of utilized ORs are transferred to idle ORs.

Denton, Rahman, Nelson, and Bailey (2006) used a discrete event simulation model for the analysis and design of an endoscopy suite to investigate different surgeon-toOR allocation scenarios. Competing performance measures such as overtime for the endoscopy suite and patient waiting time were analyzed in the model and a simple simulated annealing heuristic was used to improve the scheduled start time of cases with respect to expected overtime and patient waiting time. The endoscopy suite is a simplification of a general outpatient surgical suite since the case mix is simple (e.g. upper or lower endoscopies only) and patients see surgeons on a first-come-first-serve basis, i.e., patients are not typically assigned a specific endoscopist in advance.

In the general surgery scheduling context, Dexter, Macario, Traub, Hopwood, and Lubarsky (1999) used simulation to find an appropriate way of allocating block time to surgeons and scheduling elective cases in order to increase OR utilization. They evaluated four on-line bin packing algorithms to schedule elective cases: next fit, first fit, best fit, and worst fit. Everett (2002) described a simulation model constructed for scheduling patients waiting for surgery into the surgical suites in 4 public hospitals. Dexter, Macario, and Traub (1999) evaluated 10 different algorithms (online, off-line, and hybrid algorithms) for scheduling add-on cases into the open OR time available to evaluate their effectiveness in increasing operating room suite utilization.

Marcon, Kharraja, Smolski, Luquet, and Viale (2003) simulated a surgical suite to estimate the number of PACU beds required. They also investigated the effect of a decrease in the number of porters (patient escorts) in the OR on the number of PACU beds needed. Lowery (1992) used a simulation tool to study the effect of decreasing the number of ORs in a hospital. They analyzed the effects of changes in the surgical schedule and in case times on the number of rooms required. Tyler, Pasquariello, and Chen (2003) simulated an operating room to determine the optimum operating room utilization and analyzed the important factors such as average patient waiting time and variability of case durations which impact OR utilization. Lowery and Davis (1999) used a simulation model to simulate the patient flow through the critical care units to determine the number of beds required.

\section{SURGERY SCHEDULING BACKGROUND}

The Mayo Clinic outpatient procedure center (OPC) is a complex system with several surgical departments sharing resources. The layout of the suite is illustrated in Figure 2. It is best described as having two independent sections. There is the pre/post room area, and the operating room (OR) area. The pre/post room area consists of 20 small rooms which function as the pre procedure area and the post recovery area before and after surgery, respectively. Each room contains a small closet on wheels for the patient's personal items. During the patient's procedure, this closet can be moved out of the room so that another patient may 


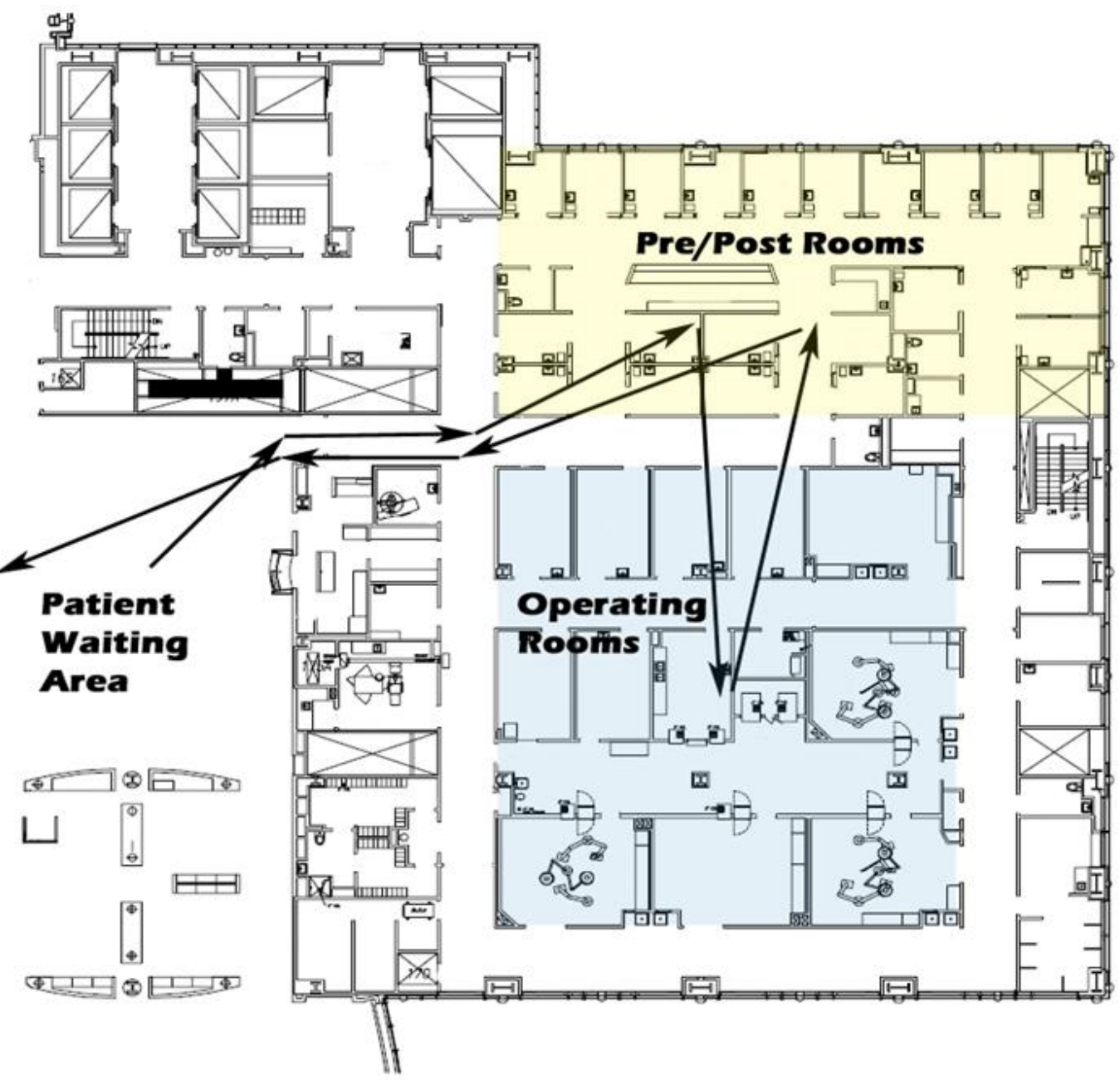

Figure 1: Illustration of the layout and patient flow through an outpatient procedure center including intake and recovery rooms, and operating rooms.

use that room if there is a shortage of rooms available. Upon completion of their procedure, the patient usually returns to their original pre/post room, or to some other open room for recovery. The flexible nature of these rooms is a recent design innovation compared to traditional surgical suites.

The OR area consists of 11 ORs with varying functions and fixed equipment, along with the core area and autoclave cleaning area where sterile instruments and supplies are stored. For the most part ORs are allocated to certain surgical areas, and therefore fixed in their utility; however, equipment used by the ORs is often standard. Prior to each days scheduled surgery, a cart with all the instruments required for the specific surgery is prepared and placed in the core area. This ensures that each procedure begins with a fully prepared set of instruments; however, the unit also retains the ability to autoclave instruments during the day, should it be necessary.

Typical patient movement through the area is to move from the patient waiting area into a pre/post room, from pre/post into a specific OR, back into pre/post for recovery, and then finally exiting from the area. For some surgical procedures, such as oral maxillofacial surgery, patients do not require a pre/post room and will enter and exit directly to/from the OR.

The outpatient procedure center has four primary departments with dedicated use of eight ORs allocated to surgical areas as follows: Pain Clinic with one, Urology and Ophthalmology with two each, and Oral Maxillofacial (OMS) with three ORs. In addition, the primary departments, with the exception of Ophthalmology, have two additional ORs which can be used during periods of high demand. There 


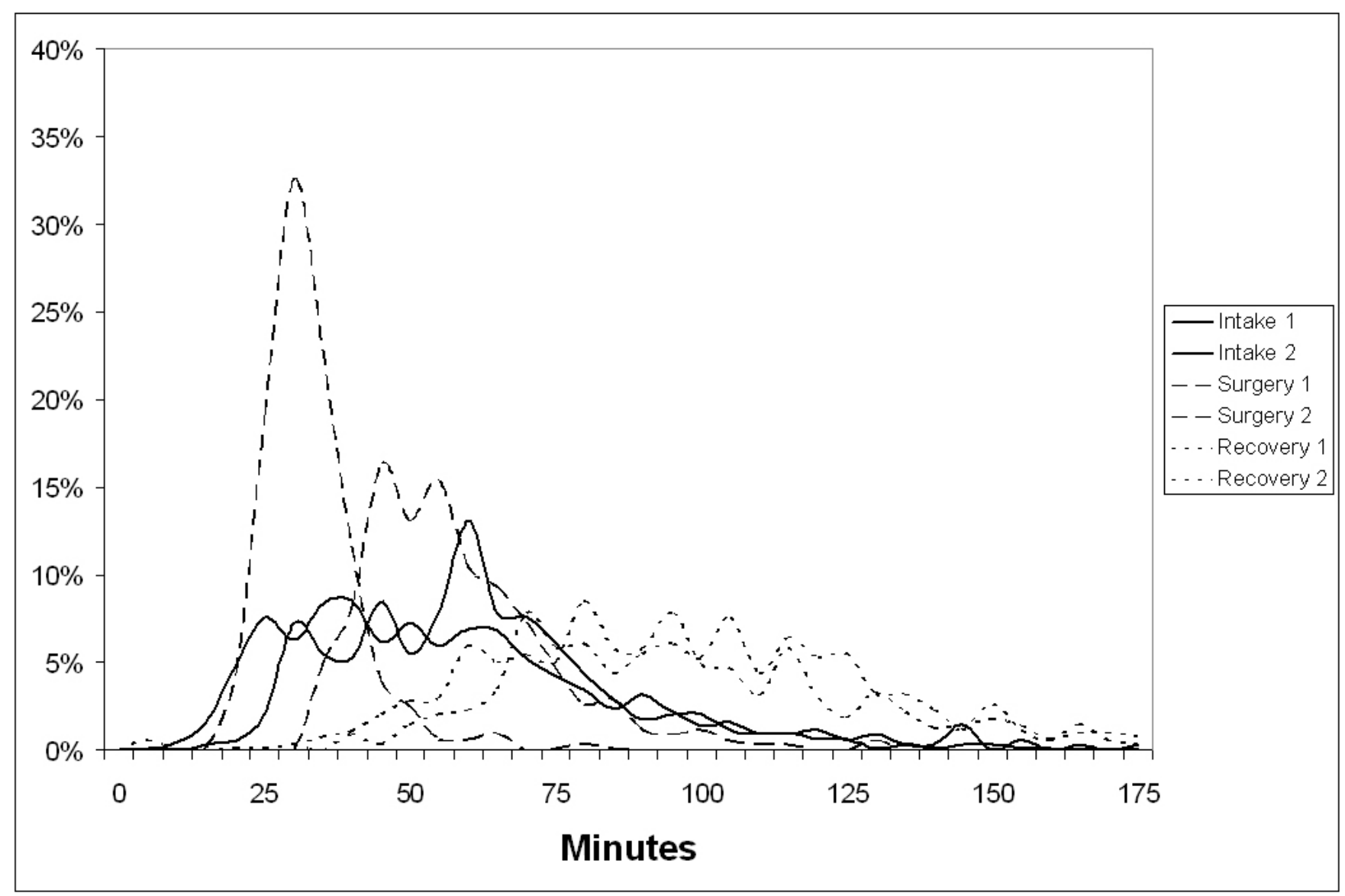

Figure 2: Probability density functions for intake, surgery, and recovery times, for two different types of surgery.

is also one additional OR which is used primarily by other departments as over-flow capacity (e.g. Dermatology). In the case of Urology and Ophthalmology, one surgeon generally uses both ORs, moving between them when needed. Having multiple ORs available allows surgeons to avoid waiting for an OR to be turned over.

Because of the mix of surgical areas, scheduling within the OPC can be a difficult process, and is subject to several factors which influence efficiency of the area. Although the departments using the OPC do not compete for OR resources, they do compete for the pre/post rooms. Thus, it may not be possible for a department to maximize its OR utilization due to constrained pre/post room capacity. This is somewhat mitigated in that pre/post rooms can be allocated to a second patient during long procedures (particularly Urology which occasionally performs cases exceeding 3 hours in length). The departments also compete for staffing resources; however, these resources are more flexible, and the layout of the area allows most staff to be used in any area. One exception is the surgeons, which are unique to the specific department they serve.

There are a number of factors that influence scheduling decisions in the OPC. For instance, due to the nature of some of the procedures, some patients are older and have underlying complications, such as diabetes. Since patients must fast prior to surgery to avoid problems with anesthesiology, those patients with diabetes are at higher risk of blood sugar problems, and are given a higher priority for early morning procedures. Additionally, older patients have a higher probability of complications, and therefore are given a higher priority. A consequence of this scheduling is that longer, more varied surgeries tend to be scheduled earlier in the morning. The OPC opens at $8 \mathrm{am}$, which is the scheduled time of patient arrival for the first surgeries of the day. The planned closure time for the OPC is $5 \mathrm{pm}$ and limiting overtime (the time beyond $5 \mathrm{pm}$ at which the last patient exits the OPC) is an important consideration in determining the number of type of surgeries that are scheduled on a given day.

\section{SIMULATION MODEL}

A discrete event simulation model of the OPC was created using Arena version 10.0. Using existing data compiled over the period of one year, probability distributions were estimated for all processes of a patient's movement through 


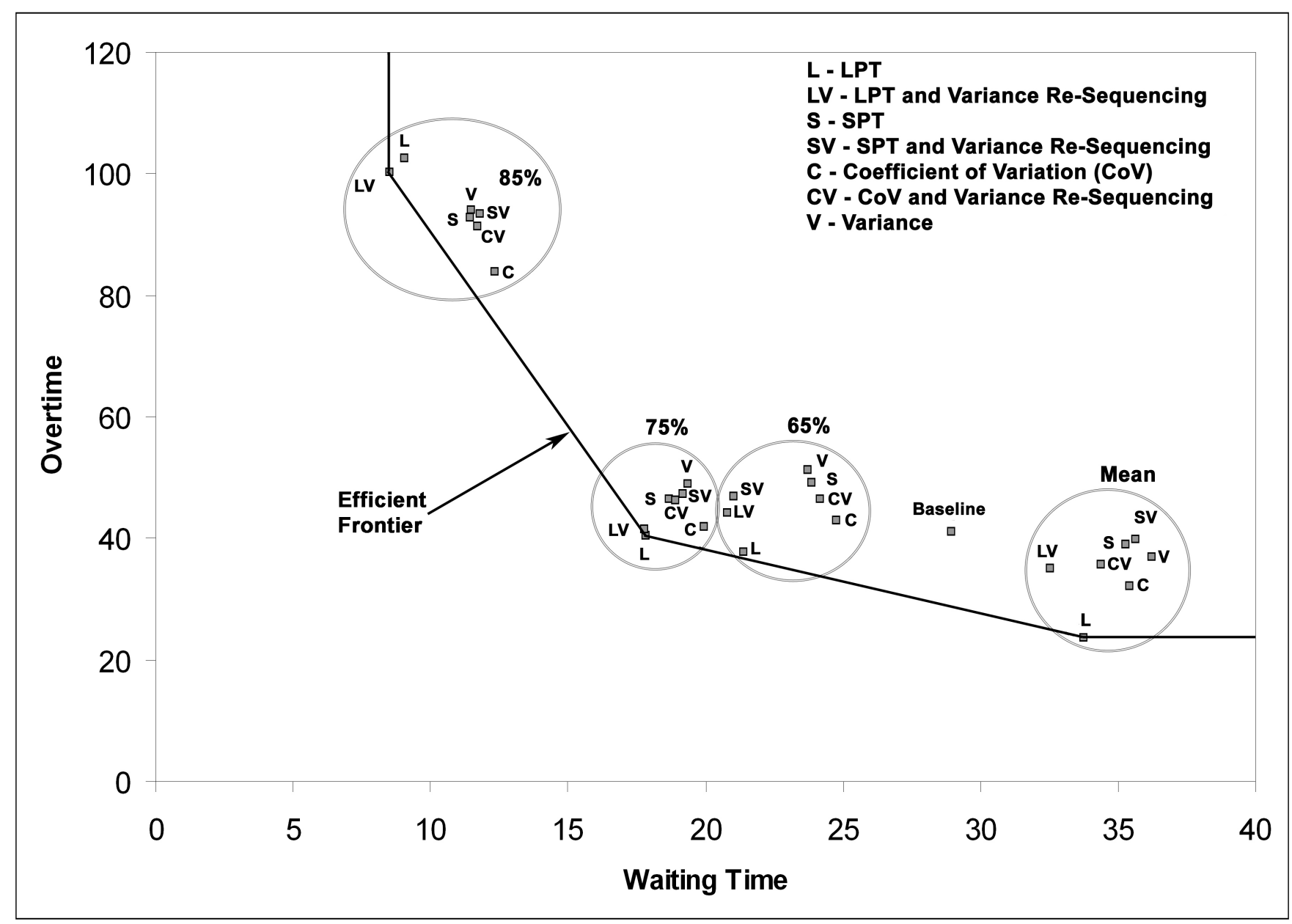

Figure 3: The efficient frontier for all combinations of sequencing and arrival time scheduling heuristics.

the OPC. These included pre-procedure, procedure, and recovery, as well as movement from one area to another. Distributions were fit with respect to procedure type, which allowed for a meaningful distribution to be estimated for most surgeries scheduled in the OPC. Primary resources for the simulation were the 11 operating rooms and 20 pre-procedure/recovery rooms. Staffing concerns, while important resources, were not considered during the validation period of the model, but may be considered in future simulations. The entities of the simulation were the patients.

Figure 2 illustrates the probability density function (pdf) for intake, procedure, and recovery time, for two different surgeries occurring in the OPC. The general form of the pdf is typical of most surgeries, in which there is a pronounced peak around the mean, and then a long tail which can be attributed to unexpected complications encountered during surgery. The intake time pdfs for the two surgeries are different, reflecting the different preparation required for different types of surgery. For instance, oral maxillofacial surgery (such as wisdom teeth extraction) requires little or no preparation prior to surgery. On the other hand, some cases require a more detailed preparation by a nurse or nurse anesthetist. Recovery time pdfs, on the other hand, are similar for most surgeries done in the OPC, since they use similar levels of anesthetic.

Distributions were fit using Arenas Input Analyzer. A total of 84 probability distributions were fit for the six departments and five processes, with most departments having multiple procedure groups. When possible, continuous distributions were estimated using actual data and consisted of Erlang, Gamma, Beta, Log Normal, Weibull, and Exponential distributions. Some processes within departments had insufficient data to accurately fit a probability distribution and Triangular distributions based on subjective estimates of minimum, mean, and maximum time were used. For instance, processes involving room turnover were modeled using Triangular distributions based off the expertise of staff working in the area.

The model is a terminating simulation, in the sense that a finite number of surgeries are scheduled each day within a pre-determined time in which the OPC is open each day. Patients that have not completed recovery by the time of 


\section{Huschka, Denton, Gul and Fowler}

planned closure of the OPC contribute to the accumulation of overtime. Patients arrive into the check-in area according to a deterministic schedule. We assume arrivals are on-time and all patients show-up for their scheduled procedure. Based on interviewing schedulers of the OPC this is a reasonable assumption.

\section{SCHEDULING HEURISTICS}

We test several combinations of sequencing, scheduling, and procedure start time heuristics for defining patient arrival times to the OPC. The scheduling heuristics define the allocation of surgical cases to ORs as follows. Each surgical specialty is considered separately in assigning surgeries to ORs. We sequence the surgeries according to increasing mean (SPT), decreasing mean (LPT), increasing variance (VAR), and increasing coefficient of variation (COV). For each heuristic we sequentially allocate surgeries to ORs such that each procedure is placed in the least utilized OR.

For each scheduling heuristic we evaluate two different sequences of surgeries in each OR. The first, is the natural sequence generated by the scheduling heuristic. Thus, for example, in the case of the LPT scheduling heuristic the sequence generated is one of decreasing mean duration of cases in the OR. The second sequence we investigate is in order of increasing variance. We elected to investigate this re-sequencing heuristic because there is evidence in the literature that such sequencing may reduce waiting time by placing highly variable surgeries at the end of the day where they can have limited impact on future patients in the schedule (Denton, Viapiano, and Vogl 2007). We evaluate the variance re-sequencing for SPT, LPT, and COV only, since the natural sequencing of VAR is in order of increasing variance.

After allocating surgeries to ORs, and sequencing the surgeries in each OR, we set patient arrival times to the OPC. The first patient is assumed to arrive at the beginning of the day, and subsequent arrivals occur at the previous patient's arrival time plus the estimated time for the previous patients procedure (since ORs tend to be the bottleneck). We determine the time allocation for procedures in four ways. The first is based on the mean duration of procedures. The remaining three alternatives use the 65 th, 75 th, and 85 th percentiles of procedure duration assuming a normal distribution while calculating percentile values. The purpose of the latter methods, known in the scheduling literature as job hedging, is to provide additional buffer time to reduce the impact on patient waiting time for surgeries running longer than the mean.

\section{EXPERIMENTATION AND PRELIMINARY RESULTS}

Validation of the discrete event simulation model was performed using actual patient arrivals into the OPC. Twentyfive replications of five months (107 days) of patient arrivals were simulated. The results were then used to validate the simulation model according to known operating room utilization rates and patient throughput. After validation, model experimentation was performed to test alternative scheduling protocols. Experimentation was done on randomly generated patient arrivals based off historical frequencies of patient types. Schedules were generated for 100 days using various scheduling techniques.

Using the OPC discrete event simulation model we have tested seven combinations of OR allocation heuristics and sequencing heuristics (LPT, SPT, VAR, COV, LPT-VAR, SPT-VAR, COV-VAR) where the latter three correspond to re-sequencing surgeries in each individual OR in order of increasing variance. We also tested four different start time heuristics: mean, 65 th, 75 th, and 85 th percentiles. We tested all combinations of allocation, sequencing and start time heuristics for a representative sample of daily surgical mixes for a 100 day period in 2006. As aggregate performance measures, for each we computed the mean patient waiting time for surgery (averaged across all patients scheduled on a given day) and the mean overtime with respect to the expected closure time $(5 \mathrm{pm})$ of the OPC. These performance measures are in conflict since mean patient waiting times are decreasing in patient inter-arrivals, while mean overtime is increasing.

Figure 3 illustrates the relative values for each of the 28 heuristics tested (L,V,C,S,LV,CV,SV are abbreviated acronyms for the sequencing heuristic and M,65,75,85 indicate the start time heuristics), including the baseline schedule which is the actual schedule used in practice. Based on Figure 3 we observe significant dispersion of the heuristics in the criteria space. The baseline schedule appears to provide some reasonable tradeoff between overtime and waiting time. However, some heuristics, such as L75 (LPT, 75th percentile), could be used to reduce mean patient waiting time with no increase in mean overtime. In general the L and LV heuristics tend to be on or very near the efficient frontier.

In general from the results in Figure 3 we observe that the start time heuristics have the most significant impact on the performance measures. The OR allocation heuristics and the resequencing heuristic have comparatively smaller effects, with the latter having very little impact on performance measures in general. The latter is counter intuitive since resequencing has been observed to have a significant impact on patient waiting time (Denton, Viapiano, and Vogl 2007). An apparent explanation for this is that the pre-procedure intake stage has substantial mean and variance of duration 

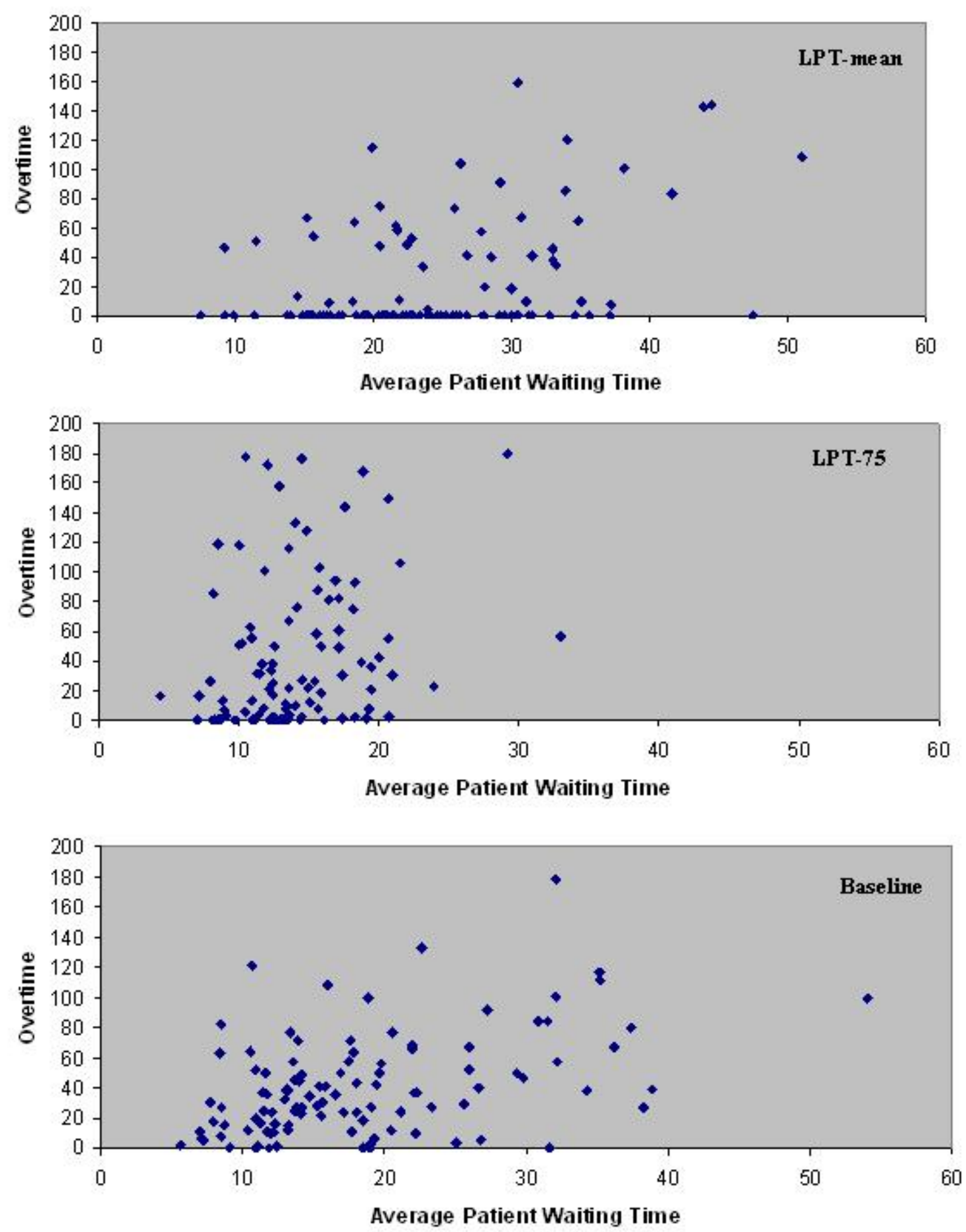

Figure 4: Comparision of 100 daily schedules for two heuristics and the baseline schedule.

relative to procedure time (e.g. see Figure 2). Thus, this high variance may reduce the effects of surgery resequencing.

Figure 4 provides greater detail on the baseline schedule, as well as two of the heuristics displayed in figure 3: LPT-mean and LPT-75. Graphs of each of the 3 types of schedules illustrate the mean waiting time and overtime for each of the 100 schedules that were tested. There is a different mix of surgeries each day and from Figure 4 it is clear that there is significant variation in performance from day to day. Thus, we conclude that the relative mix of surgeries on a given day significantly influences performance measures, and controlling the mix among days may be an 


\section{Huschka, Denton, Gul and Fowler}

opportunity to improve efficiency of the OPC and increase patient throughput.

\section{CONCLUSIONS}

Surgical services require the coordination of many activities, including patient check-in and pre-procedure preparation, the surgical procedure, and recovery. In this paper, we discussed the development of a simulation model of an outpatient procedure center. We also demonstrated that how surgeries are scheduled has an impact on the competing objectives of mean patient waiting time and the amount of overtime of the OPC. In particular, we found that arrival time schedules substantially influence expected overtime and patient waiting time, while surgery allocation and sequencing heuristics have a smaller effect. Furthermore, we find that surgery mix on a particular day is an important factor affecting performance measures, indicating that the optimization of daily surgical mix may be a promising opportunity for improving scheduling efficiency in an OPC. We will continue to use the model to study how to improve OPC operations. In addition, the model developed for the OPC has become the starting point for a model that is being used to help design a new outpatient procedure center.

\section{ACKNOWLEDGEMENTS}

This project was funded in part by grants DMI-0620573 (Denton)and DMI-0620504 (Fowler) from the National Science Foundation. We are grateful to the the staff of the Mayo Clinic OPC who were very helpful in supplying us with information for our model.

\section{REFERENCES}

Denton, B., A. Rahman, H. Nelson, and A. Bailey. 2006. Simulation of a multiple operating room surgical suite. In Proceedings of the 2006 Winter Simulation Conference, ed. L. Perrone, F. Wieland, J. Liu, B. Lawson, D. M. Nicol, and R. Fujimoto, 414-424. Piscataway, New Jersey: Institute of Electrical and Electronics Engineers, Inc.

Denton, B., J. Viapiano, and A. Vogl. 2007. Optimization of surgery sequencing and scheduling decisions under uncertainty. Health Care Manage Sci 10 (1): 13-24.

Dexter, F., A. Macario, and R. Traub. 1999. Which algorithm for scheduling add-on elective cases maximizes operating room utilization? Anesthesiology 91:14911500 .

Dexter, F., A. Macario, R. Traub, M. Hopwood, and D. Lubarsky. 1999. An operating room scheduling strategy to maximize the use of operating room block time: Computer simulation of patient scheduling and sur- vey of patients' preferences for surgical waiting time. Anesthesia and Analgesia 89:7-20.

Dexter, F., and E. Marcon. 2006. Impact of surgical sequencing on post anesthesia care unit staffing. Health Care Management Science 9:87-98.

Everett, J. E. 2002. A decision support simulation model for the management of an elective surgery waiting system. Health Care Management Science 5:89-95.

Lowery, J. 1992. Simulation of a hospital's surgical suite and critical care area. In Proceedings of the 1992 Winter Simulation Conference, ed. J. Swain, D. Goldsman, R. Crain, and J. Wilson, 1071-1078. Piscataway, New Jersey: Institute of Electrical and Electronics Engineers, Inc.

Lowery, J., and J. Davis. 1999. Determination of operating room requirements using simulation. In Proceedings of the 1999 Winter Simulation Conference, ed. P. Farrington, H. Nembhard, D. Sturrock, and G. Ewans, 1568-1572. Piscataway, New Jersey: Institute of Electrical and Electronics Engineers, Inc.

Marcon, E., S. Kharraja, N. Smolski, B. Luquet, and J. Viale. 2003. Determining the number of beds in the postanesthesia care unit: A computer simulation flow approach. Anesthesia and Analgesia 96:1415-1423.

Tyler, D., C. A. Pasquariello, and C. H. Chen. 2003. Determining optimum operating room utilization. Anesthesia and Analgesia 96:1114-1121.

\section{AUTHOR BIOGRAPHIES}

TODD R. HUSCHKA is a Master Health Systems Analyst II at Mayo Clinic in the Health Care Policy and Research department of Health Sciences Research. His primary interests are simulation modeling, optimization, and statistical analysis relating to improvements in health care systems. He completed his MS in Industrial Engineering Decision Science/Operations Research at the University of Wisconsin, Madison, Wisconsin. His email address is 〈todd.huschka@mayo. edu〉.

BRIAN T. DENTON is an Assistant Professor at North Carolina State University in the Edward P. Fitts Department of Industrial and Systems Engineering. Previously he was a Senior Associate Consultant at Mayo Clinic in the College of Medicine. His primary research interests are in the development and application of operations research methods to health care delivery, and his work relating to surgical scheduling won the IIE Transactions outstanding publication award in 2005. He completed his Ph.D. in Management Science at McMaster University in Hamilton, Ontario, Canada. His email address is bodenton@ncsu.edu〉. 
SERHAT GUL is a PhD candidate and a research assistant in the Industrial Engineering department at Arizona State University. His primary research interests are in operating room scheduling and simulation. He completed his BS in Manufacturing Systems Engineering at Sabanci University, Istanbul, Turkey His email address is $\langle$ serhat . gul @asu . edu〉.

JOHN W. FOWLER is a Professor in the Operations Research and Production Systems group of the Industrial Engineering Department at Arizona State University. His research interests include modeling, analysis, and control of manufacturing and service systems. He is a Fellow of the Institute of Industrial Engineers and is the SCS representative on the Board of Directors of the Winter Simulation Conference. He is an Area Editor of the Transactions of the Society for Computer Simulation International, an Associate Editor of IEEE Transactions on Semiconductor Manufacturing, and on the Editorial Board of IIE Transactions. His email address is 〈john. fowler@asu.edu〉. 